\title{
Kelvin-Helmholtz and Holmboe instabilities of a diffusive interface between miscible phases
}

\author{
T. Zagvozkin* \\ Institute of Continuous Media Mechanics UB RAS, Perm, Russia \\ A. Vorobev $^{\dagger}$ \\ University of Southampton, Faculty of Engineering and Physical Sciences, UK \\ T. Lyubimova \\ Institute of Continuous Media Mechanics UB RAS, Perm, Russia and \\ Perm State University, Russia
}

(Dated: June 10, 2019)

\begin{abstract}
The stability of a shear flow imposed along a diffusive interface that separates two miscible liquids (a heavier liquid lies underneath) is studied using direct numerical simulations. The phase-field approach is employed for description of a thermo- and hydrodynamic evolution of a heterogeneous binary mixture. The approach takes into account the dynamic interfacial stresses at a miscible interface and uses the extended Fick's law for setting the diffusion transport (the diffusion flux is proportional to the gradient of chemical potential). The shear flow is unstable to two kinds of instabilities: (i) the Kelvin-Helmholtz instability, with an immovable vortex formed in the middle of an interface (in the vertical direction), and (ii) the Holmboe instability, with travelling waves along the interfacial boundary. The development of the Holmboe instability results in a stronger enhancement of molecular mixing between the mixture components. Earlier, the boundaries of these instabilities were determined using the linear stability analysis and employing the concept of a 'frozen interface'. In the current work, through the solution of full equations, we obtain the stability boundaries for several sets of governing parameters, showing a greater variety of the possible shapes of the stability diagrams. The Kelvin-Helmholtz instability always occurs at lower gravity effects (lower density contrasts), while the Holmboe instability occurs when gravity is stronger. We show that for some parameters these two instabilities are separated by a zone where the shear flow is stable, and this zone disappears for the other sets of parameters.
\end{abstract}

\section{INTRODUCTION}

Mixing in liquid/liquid or gas/liquid systems can be intensified by taking advantage of hydrodynamic instabilities, e.g. the instabilities induced by a shear flow. In the current work we investigate the effects of a shear flow on the mixing of two miscible liquids that are initially separated by a thin horizontal interface, and with a heavier liquid lying underneath. We assume that the liquids are just brought into contact, so the initial thermodynamic state of a mixture is different from the state of thermodynamic equilibrium, which induces the interfacial diffusion. The resultant simultaneous thermo- and hydrodynamic changes in a heterogeneous binary mixture are examined.

It is well known that the shear flow in a homogeneous fluid is unstable due to the Kelvin-Helmholtz instability $[1,2]$. This instability was studied in numerous works, and the major results are summarised in books $[3,4]$. In the current work, we consider the shear flow in a inhomogeneous fluid. Namely, the shear flow is enforced along the interface of two liquids of different densities. It is

\footnotetext{
* timofey@zagvozkin.ru

† A.Vorobev@soton.ac.uk

‡ lubimova@psu.ru
}

known that the density stratification affects the development of the Kelvin-Helmohotz instability.

The density stratification is traditionally determined by the Richardson number $R i=N^{2} / U^{2}$, where $U$ is the local mean velocity and $N$ is the Väisälä frequency,

$$
N(y)=\sqrt{-\frac{g}{\rho} \frac{d \rho}{d y}} .
$$

In this formula, $\rho$ is the fluid density, $g$ is the gravity acceleration, and $y$ is the vertical coordinate. Miles and Howard [5] found that the Kelvin-Helmholtz instability in a density-stratified medium does not develop if the density stratification is relatively strong, namely, when $R i>1 / 4$ everywhere. Holmboe [6] however showed that if the thickness of a density profile is much smaller than the thickness of a shear flow profile, then, despite the strong density stratification, the flow is unstable due to a new instability that develops through the growth of two travelling waves in the layer. Thus, in a density stratified medium the shear flow may become unstable to both the Kelvin-Helmholtz and Holmboe instabilities.

In a number of later studies the boundaries of the Kelvin-Helmholtz and Holmboe instabilities were determined for various density and velocity profiles [7-13], including the cases when the central points of the density and velocity profiles were not coincident [14, 15]. 
The influence of other complexities, such as surface tension [16], larger density contrasts [17, 18], and diffusion $[19,20]$ were also examined. The existence of both Kelvin-Helmholtz and Holmboe instabilities were also experimentally confirmed [21-23].

In the current work the evolution of a heterogeneous binary mixture is traced on the basis of the phase-field approach. This approach represents an interface as a transitional layer of a finite thickness, with all variables, including density, experiencing sharp but continuous changes across the interfacial boundary. By imposing a shear flow along the interface we obtain a classical problem of stability of a shear flow in a density stratified medium. However, in contrast with other studies the use of the phase-field approach allows an accurate description of a multi-phase system. Namely, the approach takes into account the effects of interfacial stresses that should be also associated with miscible boundaries [24, 25]. In addition, the approach takes into account that the classical Fick's law is only applicable for lower concentration gradients and thus the Fick's law is not strictly valid for liquid/liquid interfaces when the concentration gradients are large. The phase-field approach is based on the extended Fick's law that states that the diffusion flux is proportional to the gradient of chemical potential. This extension, in particular, allows for the convenient description of the interfacial diffusion in partially miscible liquids that are miscible until saturation levels are reached in neighbouring phases (which is an obvious case when the Fick's law fails, as in this case there is a strong concentration gradient across the interface but there is no interfacial diffusion). In addition, this extension allows for the account of barodiffusion effect.

All these features make the problem studied in our work different from the other studies of the KelvinHelmholtz and Holmboe instabilities. In the current work the development of the classical hydrodynamic instabilities occurs in a two-phase system with an undergoing phase transition, while in other studies the development of the instabilities happen either in a single-phase stratified medium, or in a two-phase immiscible system.

The linear stability of a phase boundary in a heterogeneous binary system is investigated in work [26], where the boundaries of the Kelvin-Helmholtz and Holmboe instabilities are determined. It is found that the zones of instability are larger for thinner interfaces. The zones of instability are also enlarged by diffusive and capillary effects. Viscosity is found to play its usual stabilising role. The linear stability analysis is based on the the assumption of a 'frozen interface', i.e. assuming that the diffusive smearing of the interface occurs slowly, much slower than the growth of the hydrodynamic perturbations. The aim of the current work is to verify the linear analysis by solving the full non-linear equations, to understand the differences in the flow fields that characterise these two different instabilities, and to extend our previous findings by examining the stability of the shear flow for greater variety of governing parameters.

\section{MATHEMATICAL MODEL}

The idea of the phase-field approach is to apply one system of the governing equations to determine the flow fields in a whole multiphase system, including interfaces. The interfaces are represented by transitional layers of a finite thickness. The position of interfaces is determined from the field of concentration, namely, the interfaces correspond to the places with larger concentration gradients. To take into account the surface tension effects that are associated with interfacial boundaries, the free energy function of a mixture is re-defined by adding a new gradient term [27],

$$
f(C, \nabla C)=f_{0}(C)+\frac{\epsilon}{2}(\nabla C)^{2} .
$$

Here, $f$ is the specific free energy function of a binary mixture, $f_{0}$ is its classical part, and $\epsilon$ is the capillary constant that defines the strength of the capillary effects. The capillary constant is usually so small that the second term is negligible everywhere except for the places with large concentration gradients, i.e. except for the interfaces.

The full hydrodynamic equations for a binary system that is defined by free energy function (2) were derived by Lowengrub and Truskinovsky [28]. The full equations are however too hard for direct numerical simulations, as these equations include the full continuity equation (that is called the effect of quasi-compressibility and that is explained by the dependence of the mixture density on concentration). It was later shown that the slower convective and diffusive evolution of a mixture can be determined on the basis of the fully incompressible equations, that represent the Boussinesq approximation of the full Cahn-Hilliard-Navier-Stokes equations [29, 30]. The Boussinesq approximation of the Cahn-Hilliard-NavierStokes equations are numerically solved in the current work to describe the evolution of a heterogeneous binary mixture.

The governing equations include the equations for conservation of momentum, species, and mass,

$$
\begin{gathered}
\frac{\partial \vec{u}}{\partial t}+(\vec{u} \cdot \nabla) \vec{u}=-\nabla \Pi+\frac{1}{R e} \nabla^{2} \vec{u}-C \nabla \mu, \\
\frac{\partial C}{\partial t}+(\vec{u} \cdot \nabla) C=\frac{1}{P e} \nabla^{2} \mu, \\
\nabla \cdot \vec{u}=0 .
\end{gathered}
$$

Here $\vec{u}$ is the fluid velocity, $\Pi$ is the modified pressure that is to be determined from an incompressibility constraint, $t$ is the time, $C$ is the concentration that is defined as the mass fraction of one of the components in a mixture, and $\mu$ is the chemical potential. The Navier-Stokes equation (3) includes an additional term (frequently called the Korteweg force) that takes into account the surface tension 
effects on a liquid/liquid interface. The diffusion process is driven by the gradient of the chemical potential that is defined by the following expression,

$$
\mu=G r(\vec{r} \cdot \vec{\gamma})+\frac{d f_{0}}{d C}-C n \nabla^{2} C .
$$

The classical part of the free energy function, $f_{0}$, is chosen so to define the expected thermodynamic behaviour of a binary mixture. In this work, we study the evolution of a heterogeneous binary mixture with undergoing phase transformations. In particular, we consider a binary mixture with the upper critical solution temperature (that defines the position of the so-called consolute point), when the components of a mixture are miscible in all proportions above the critical temperature, and only partially miscible (up to a certain solubility level) if the mixture temperature is below the critical value. This is the most popular type of the phase behaviour among all binary mixtures. The thermodynamic behaviour of such a mixture may be set by the Landau formula [31],

$$
f_{0}=A C^{2}+C^{4} .
$$

Another choice of the free energy function is given by the 'regular solutions' function (also known as the FloryHuggins theory) that is frequently used for setting the thermodynamic behaviour of polymer solutions [25, 27, $32]$,

$$
\begin{aligned}
f_{0}=\left(A-\frac{3}{2}\right) C^{2} & +\frac{3}{4}\left(\frac{1}{2}+C\right) \ln \left(\frac{1}{2}+C\right) \\
& +\frac{3}{4}\left(\frac{1}{2}-C\right) \ln \left(\frac{1}{2}-C\right),
\end{aligned}
$$

Here, for simplicity, the original definition of concentration, as the mass fraction of one of the components in a mixture and that varies in the interval of [0..1], is modified by a transition, $C \rightarrow\left(C-C_{c}\right)$. In addition, for simplicity, we assume that the phase diagram is symmetrical about the consolute point and $C_{c r}=1 / 2$. As a result, the range of the modified concentration is $[-1 / 2 . .1 / 2]$.

Figure 1a depicts the shapes of functions (7) and (8) and figure $1 \mathrm{~b}$ depicts the shapes of the phase diagrams, that define equilibrium states of a mixture, and that are obtained on the basis of functions (7) and (8). One sees that the consolute point of a mixture is determined by the coordinates $(A=0, C=0)$. A mixture is always homogeneous in equilibrium when $A>0$, and a mixture may be either homogeneous or heterogeneous (that is defined by the overall mass balance) when $A<0$. Hence, the non-dimensional parameter $A$ that appears in formulae (7) and (8) plays the role of the mixture temperature. This parameter is defined as $A=a / b$, where $a$ and $b$ are the two standard phenomenological parameters of the Landau theory for near-critical systems [31].

Both functions (7) and (8) produce quite similar phase diagrams, and both phase diagrams reproduce features of the experimentally expected thermodynamic behaviour, see e.g. the phase diagram of isobutyric acid/water mixture in Ref. [33]. Thus, from the point of view of matching the experimental behaviour, both functions can be successfully used.

Nevertheless, primarily owing to computational reasons, we select function (8) for the current study. In this work, the range of concentrations, $[-1 / 2 . .1 / 2]$, corresponds to the interface of physically relevant values of concentrations. In figure 1 one sees that function (8) coincides with the Landau function near the critical solution point, and this function defines a different behaviour when $|C| \rightarrow 1 / 2$, where overshooting (non-physical values in the concentration field) are excluded by the logarithmic terms. Free energy function (8) was previously used in our other works where evolving heterogeneous binary systems were examined in the frameworks of other physical problems [34-38].

The above equations are written in the nondimensional form. The following scales were used to nondimensionalise the equations,

$$
L_{*}=h, \tau=\frac{L_{*}}{u_{*}}, u_{*}=\mu_{*}^{1 / 2}, \mu_{*}=b, \Pi_{*}=\rho_{*} \mu_{*} .
$$

Here $h$ is the height of the layer, $\tau, u_{*}, \mu_{*}$ and $\Pi_{*}$ are the time scale, velocity scale, the scales of the chemical potential, and pressure; $\rho_{*}$ is the typical density that can be defined as the density of one of components of the mixture.

The governing equations include the following nondimensional parameters,

$$
\begin{aligned}
P e=\frac{\rho_{*} L_{*}}{\alpha \mu_{*}^{1 / 2}}, R e=\frac{\rho_{*} \mu_{*}^{1 / 2} L_{*}}{\eta_{*}}, G r & =\phi \frac{g L_{*}}{\mu_{*}}, \\
C n & =\frac{\epsilon}{\mu_{*} L_{*}^{2}} .
\end{aligned}
$$

These are the Peclet, Reynolds, Grashof, and Cahn numbers. We use the standard names for the first three parameters, although they are defined through nonstandard phenomenological parameters that are introduced within the phase-field approach. These parameters appear in front of the corresponding terms of the hydrodynamic equations and play similar roles in dynamic similarity of different flows.

The Peclet number sets the relative importance of diffusive effects. The coefficient $\alpha$ is called the mobility coefficient, and its value can be estimated as $\alpha=\rho_{*} D / \mu_{*}$, where $D$ is the standard diffusion coefficient. We assume that the Peclet number is always large, which means that diffusion is relatively weak, at least, on short time periods.

The Reynolds number sets the importance of the viscous force. For simplicity we assume that the difference in the viscosity coefficients of the mixture components is not strong, so that the use of one Reynolds number is sufficient.

The Grashof number sets the importance of the gravity term. We assume that the density contrast $\phi=$ 


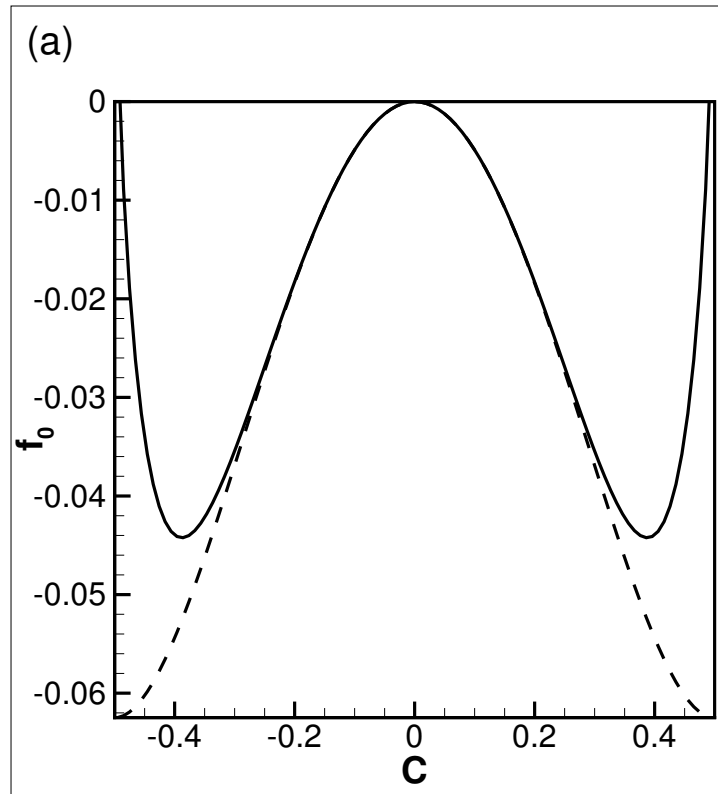

(b)

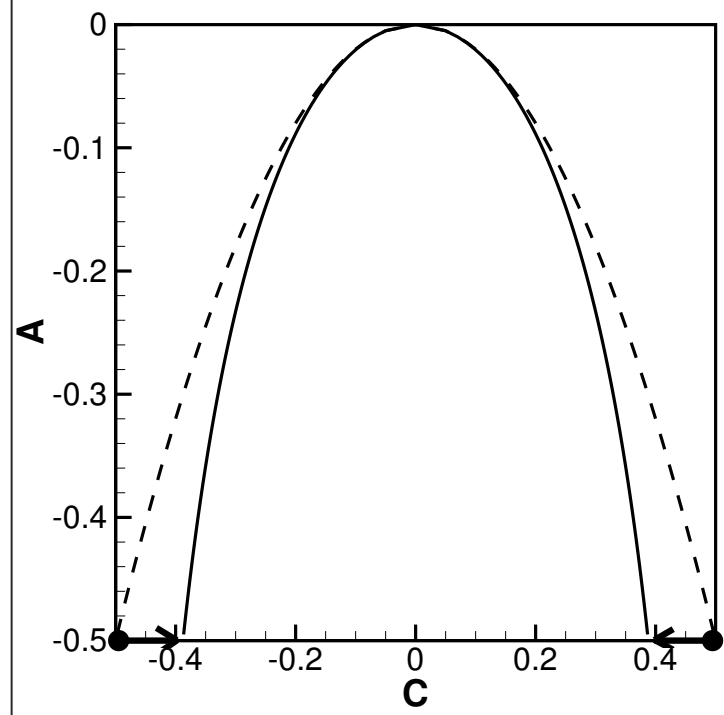

FIG. 1. The classical part of a free energy function (a) and the shapes of the phase diagrams (b) given by formulae (8), solid lines, and (7), dashed lines. The dots in (b) indicate the initial state of the mixture, and the arrows indicate the directions of the thermodynamic transformations experienced by the mixture in the current work.

$\left(\rho_{2}-\rho_{1}\right) / \rho_{*}$ is small, which is true for all liquid/liquid mixtures. The gravity term enters the governing equations though the definition of the chemical potential (6). Substitution of the chemical potential into the NavierStokes equation (3) would generate a standard convective force. Substitution of the chemical potential into the equation for the species transport (4) would cancel the gravity term. Nevertheless, the effect of the gravity in diffusion (barodiffusion) still remains, and would enter the problem through the boundary condition. To exclude the diffusive transport through the wall one needs to set zero value of the normal derivative of the chemical potential at the wall, which brings the gravity term. This effect in particular is responsible for equilibrium stratification in a mixture when the concentration of a heavier component grows towards the bottom of a layer.

The Cahn number sets the strength of the capillary forces. It also sets the equilibrium thickness of the interface that is determined as $\delta_{e q}=\sqrt{-C n / A}[28,39]$.

One additional non-dimensional parameter in equations (3)-(6) is $A$ that was introduced above and that sets the 'temperature' of a mixture (the thermodynamic behaviour of a mixture).

The governing equations are supplemented with the boundary conditions. Normally, at the rigid walls one sets the velocity vector to be zero (the no-slip condition), the normal derivative of the chemical potential to be zero (no diffusive flux through walls), and the normal derivative of concentration to be zero (the neutral wetting conditions, i.e. the contact line is orthogonal to the wall).

\section{PROBLEM STATEMENT}

We present the results of the $2 \mathrm{D}$ direct numerical modelling of the Kelvin-Helmholtz and Holmboe instabilities in a system composed of two miscible liquids that are initially separated by a flat horizontal interface. The mixture fills in a horizontal plane layer. We assume that the liquids are just brought into contact, the initial thermodynamic state of the mixture is different from the state of thermodynamic equilibrium, which induces the process of interfacial diffusion. The lighter liquid is placed on top of the denser one. The shear flow is additionally imposed along the interface. The mixture is assumed to remain isothermal.

Thus, the heterogeneous binary mixture is enclosed within a rectangular computational domain. The stability of the liquid/liquid interface with respect to an one-mode harmonic perturbation, characterised by the wavenumber $k=2 \pi / \lambda$, is studied. The periodic boundary conditions are imposed in a horizontal direction. The horizontal size of the domain is chosen to be equal to the wavelength, $\lambda$, of the initial perturbation, and thus it varies for different runs. The vertical size of the layer is used as the length scale. The horizontal and vertical coordinates are denoted by $x$ and $y$, respectively.

The initial concentration profile is set by the expression

$$
C_{0}(x, y)=0.495 \tanh \left(\frac{y-0.5(1+0.1 \cos (k x))}{\delta_{0}}\right)
$$

Here 0.495 are the initial concentrations in the two liquids in contact, and $\delta_{0}$ is the initial interface thickness. The amplitude of a perturbation is 0.1 for all runs. 
The externally imposed flow along the interface is set by the following profile

$$
U(y)=U_{0} \tanh \left(\frac{y-0.5}{\delta_{U}}\right) .
$$

Here $U_{0}$ and $\delta_{U}$ are the amplitude and thickness of the velocity profile.

In the current work, the thicknesses of the concentration and velocity profiles are treated as two independent parameters. Since we consider the evolution of a thermodynamically non-equilibrium binary mixture the initial thickness of the concentration profile is taken different from the thickness of an equilibrium interface, that is $\delta_{e q}=\sqrt{-C n / A}$.

The total vector of velocity is split into the background and perturbation parts, $\vec{u}=U \vec{i}+\vec{v}$ (the unit vector $\vec{i}$ that defines the direction of the $x$-axis also defines the direction of the imposed shear flow). The governing equations (3)-(6) are supplemented with the periodic boundary conditions in the horizontal direction, and with the following conditions at the bottom and upper plates,

$$
y=0,1: \frac{\partial v_{x}}{\partial y}=0, v_{y}=0, \frac{d \mu}{d y}=0, \frac{d C}{d y}=0 .
$$

Thus, we impose the no-stress condition for the $x$ component of the velocity, and the no-penetration condition for the $y$-component of the fluid velocity. The boundary conditions for the chemical potential and concentration assume the absence of the diffusive flux through the walls, and the condition of neutral wetting. In the current work we are interested in the development of the instabilities near the interface, which is in the middle of a layer, far from the walls. The conditions at the walls are less important for this study, and the boundary condition (14) were chosen so to minimise their influence on the flows in the bulk layer.

For the numerical solution, equations (3-6) are rewritten in the streamfunction $\left(v_{x}=\partial \psi / \partial y\right.$ and $v_{y}=$ $-\partial \psi / \partial x)$-vorticity $\left(\omega=\left(\partial v_{x} / \partial y-\partial v_{y} / \partial x\right)\right)$ formulation:

$$
\begin{gathered}
\frac{\partial \omega}{\partial t}+J(\psi, \omega)=\frac{1}{R e}\left(-U^{\prime \prime \prime}+\nabla^{2} \omega\right)+J(\mu, C), \\
\frac{\partial C}{\partial t}+U \frac{\partial C}{\partial x}+J(C, \psi)=\frac{1}{P e} \nabla^{2} \mu, \\
\nabla^{2} \psi=-\omega, \\
\mu=G r \cdot y+\frac{3}{4} \ln \left(\frac{1 / 2+C}{1 / 2-C}\right)-(3-2 A) C-C n \nabla^{2} C .
\end{gathered}
$$

Here, $J \equiv \partial / \partial x-\partial / \partial y$, and prime stands for the derivative in respect with $y$. The periodic boundary conditions are imposed in the $x$ direction, and on the walls we set,

$$
\psi=0, \frac{\partial \psi}{\partial y}=\frac{\partial \mu}{\partial y}=\frac{\partial C}{\partial y}=0 .
$$

The resultant set of equations and boundary conditions are solved using the finite-difference method on a uniform mesh. The explicit first order in time and second-order in space discretisation scheme is used.

\section{NUMERICAL RESULTS}

To choose the optimal numerical resolution we perform several numerical runs using the grids with the different numbers of computational nodes. The results of these runs are depicted in figure 2. We plot the time dependencies of the kinetic energy of a perturbation,

$$
E_{k}=\frac{1}{2} \int_{V} v^{2} d V
$$

(here $V$ is the volume of the computational domain), and the time dependencies of the length of an interfacial boundary for two different amplitudes of the externally imposed flow $U_{0}=0.2$ (figure $2 \mathrm{a}, \mathrm{b}$ ) and $U_{0}=0.4$ (figure 2c,d). One sees that perturbations decay for the lower amplitude of the shear flow and perturbations grow when the externally imposed flow is stronger.

In figure 2, the results obtained with the use of different meshes converge (the distance between the curves decreases upon the gradual improvement of the numerical resolution). In a stable case, the curves remain close to each other during the entire numerical run, until a perturbation completely decays. In an unstable case, the curves obtained with the use of different grids eventually diverge from each other. Although, as one sees in figure $2 \mathrm{c}, \mathrm{d}$, for the set of parameters used to generate this figure, the curves with two best resolutions remain quite close at least until $t \leq 10$. We then assume that the resolution with the grid size of $1 / 500$ is sufficient to produce the accurate results for an initial evolution of a mixture, $t \leq 10$, and a better resolution would be needed for tracing the growth of a perturbation on a longer time interval. Although, the improved resolution can be required for thinner initial profiles of the concentration and velocity fields, and for higher Peclet numbers (when diffusive skin-layers become thinner) and lower Cahn numbers (as this parameter sets the equilibrium thickness of the interface).

As discussed in the introductory section, there are two instabilities that may develop in the layer. Figures 3 and 4 show the snapshots that illustrate the typical developments of the Kelvin-Helmholtz and Holmboe instabilities, respectively. For the Kelvin-Helmholtz instability, one observes the development of a vortex in the middle of the layer (right in the middle of the interface). The size of the vortex and the amplitude of the flow velocity increase with time, although the horizontal location of the vortex always remains the same. In the case of the Holmboe instability, one observes the development of travelling waves on the interface and the formation of vortices that move along the layer. These simple differences (whether vortices are stationary or moving and 


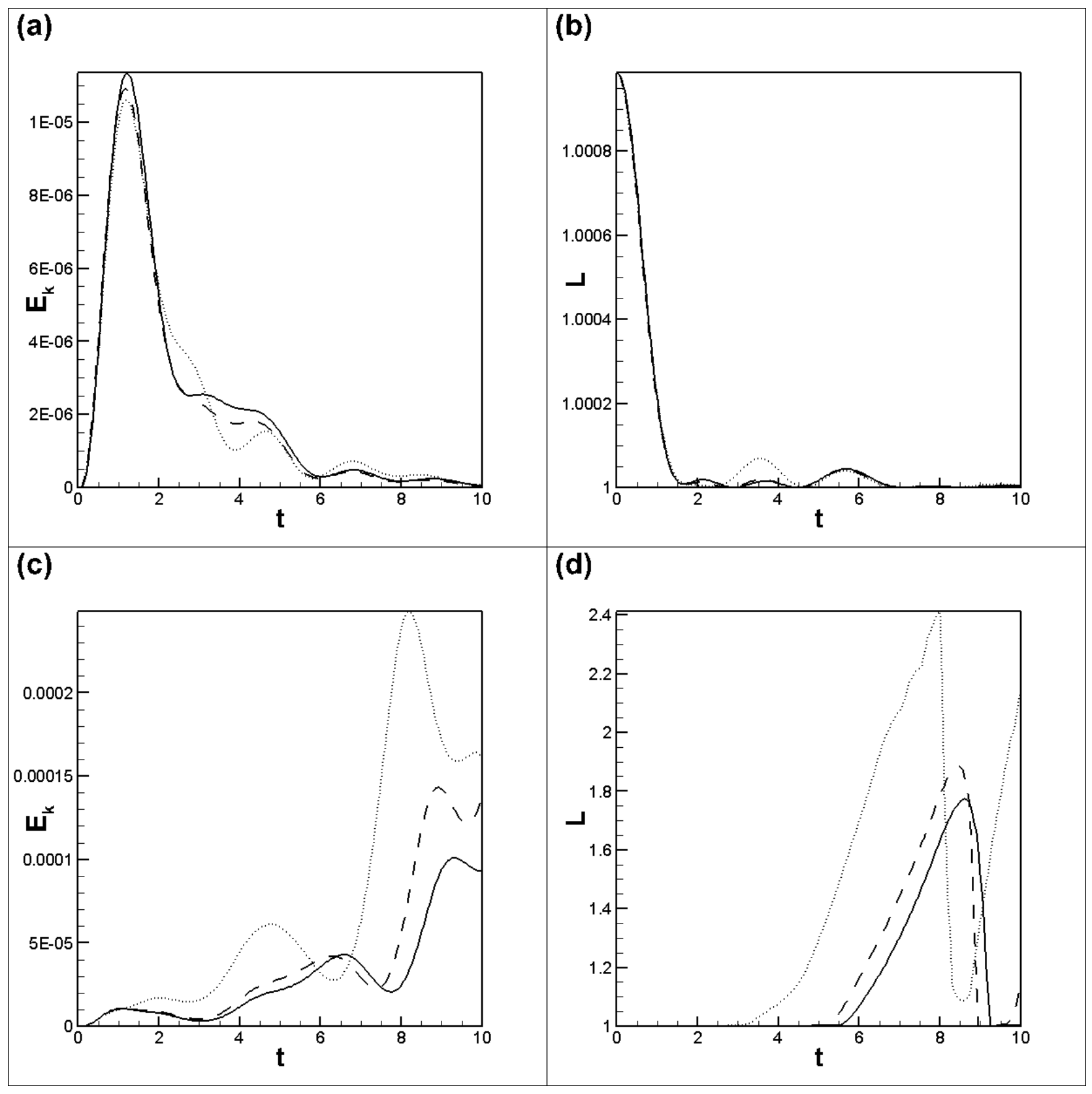

FIG. 2. The time dependencies of the total kinetic energy (a,c) and of the length of interface (b,d) are plotted for the numerical runs performed with the use of different grids, with $250 \times 250$ (dash-dotted lines), $500 \times 500$ (dashed lines), and $750 \times 750$ (solid lines) nodes. The other parameters for these runs were $k=6.28, P e=10^{6}, C n=4 \cdot 10^{-4}, \delta_{0}=\delta_{U}=0.028$, and $U_{0}=0.2(\mathrm{a}$, b) and $U_{0}=0,4(\mathrm{c}, \mathrm{d})$.

whether the interface is flat or wavy) allow us to distinguish the instabilities, and to associate the governing parameters with the particular kind of instability.

Next we want to obtain the stability diagrams to show the boundaries of the Kelvin-Helmholtz and Holmboe instabilities. For this end, we perform the series of numerical runs and calculate various integral characteristics. Namely, we calculate

- the kinetic energy of perturbations using equation (20);
- the length of the interface, $L$ (The position of the interface is determined by the concentration level $C=0$. To determine the length of the interface, $L$, we first search for the nodes between which the concentration changes its sign, and determine the position of the interface between these nodes by using the linear interpolation; we next obtain the length of an interface element that lies within a cell made of four nodes; and finally we sum up these elementary lengths to obtain the length of the whole 


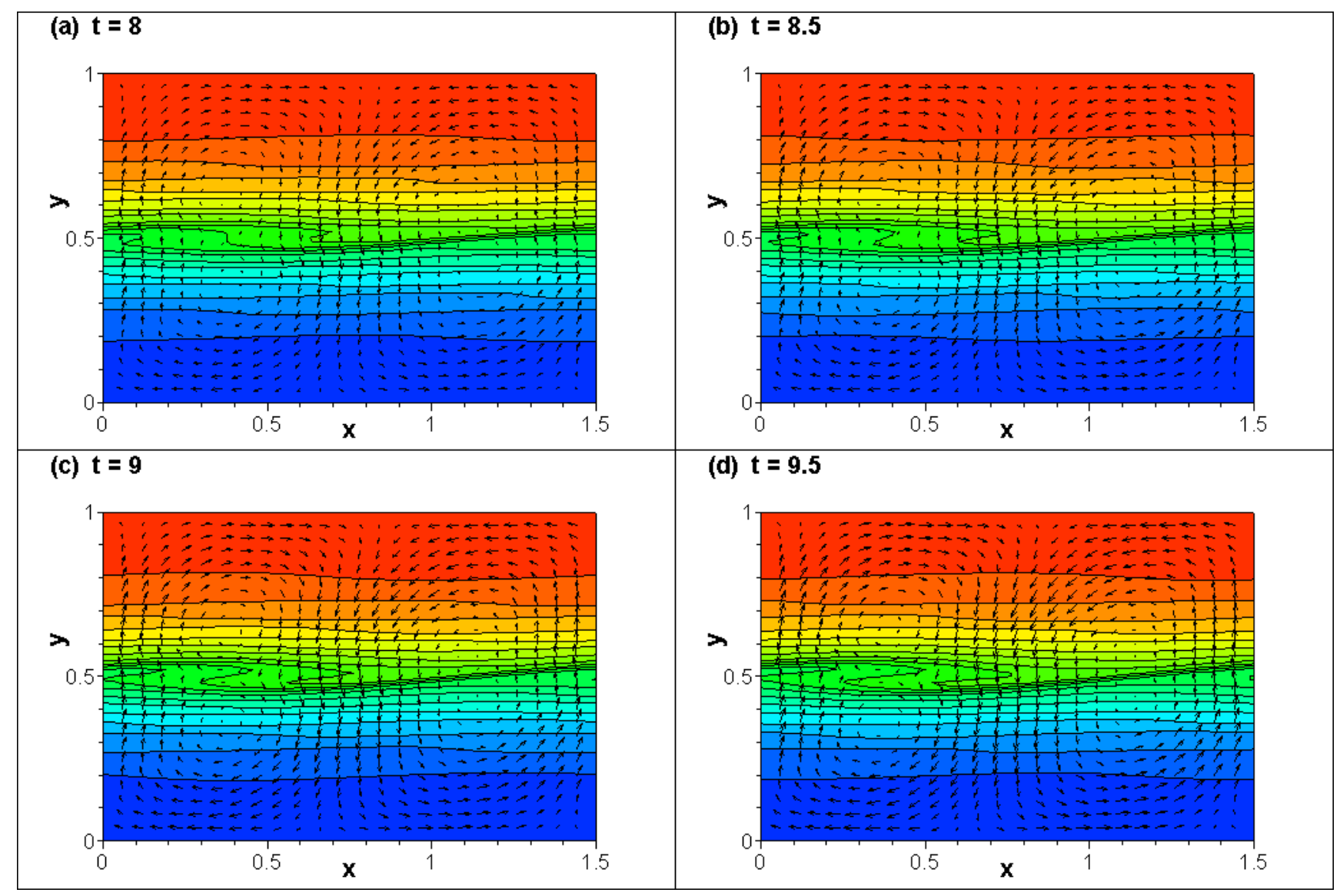

FIG. 3. Development of the Kelvin-Helmholtz instability. The fields of concentration (isolines) and velocity (vectors) at different time moments. The data are obtained for $\delta=\delta_{U}=0.2, U_{0}=1, R e=100, C n=10^{-3}, P e=10^{6}, G r=0.5, k=4.19$, resolution $750 \times 500$ nodes.

interfacial line);

- the thickness of the interface $\delta$ using the formula $\delta=V_{\delta} / L$ (here $V_{\delta}$ is the volume of the transitional zone that is defined as the region in the computational domain with the concentration levels in the range, $|C| \leq 0.2)$;

- the coefficient of the surface tension $\sigma=E_{i} / L$ (here $E_{i}$ is the interfacial energy that is calculated as $\left.E_{i}=C n \int_{V}(\nabla C)^{2} d V\right)$;

- the average concentrations in each phase, $C_{1}$ and $C_{2}$ (the phases are distinguished by the sign of the concentration; the average concentration in the part of the computational domain with positive concentrations is denoted as $C_{1}$, and the average concentration in the other part of the computational domain is $C_{2}$ ).

Figure 5 depicts two typical time evolutions of the integral characteristics. Namely, the curves are obtained for the different levels of the Grashof number, when the Kelvin-Helmhotz (solid lines) and Holmboe instabilities (dashed lines) develop. The curves demonstrate the clear differences in the time changes of the integral characteristics for these two instabilities.

For the Kelvin-Helmholtz instability, the kinetic energy of a perturbation (that has a form of a single stationary vortex) grows monotonically. For the case of the Holmboe instability, the oscillatory growth of the kinetic energy is observed, which is explained by travelling vortices. Indeed, in figure 4 , one sees that the velocity field in the computational domain is strongly different at different time moments, and this explains the strong changes (oscillations) in the values of the kinetic energy.

As stated above, the position of an interface is defined by a level of concentration, $C=0$, which is depicted by the central isoline in both figures 3 and 4 . This definition is not very accurate for rather diffusive interfaces considered in this work. Nevertheless, in figure 3 one sees that the central isoline becomes quite disturbed by the KelvinHelmholtz vortex, which explains the observed growth of the interface length, $L$, in figure $5 \mathrm{~b}$ (solid line). In figure 4, in the case of the Holmboe instability, the length of the central isoline remains almost undisturbed, which is confirmed by a shape of the dashed line in figure $5 \mathrm{~b}$.

Figure $5 \mathrm{c}$ depicts the time changes of the interface thickness. The interfacial stresses are characterised by 


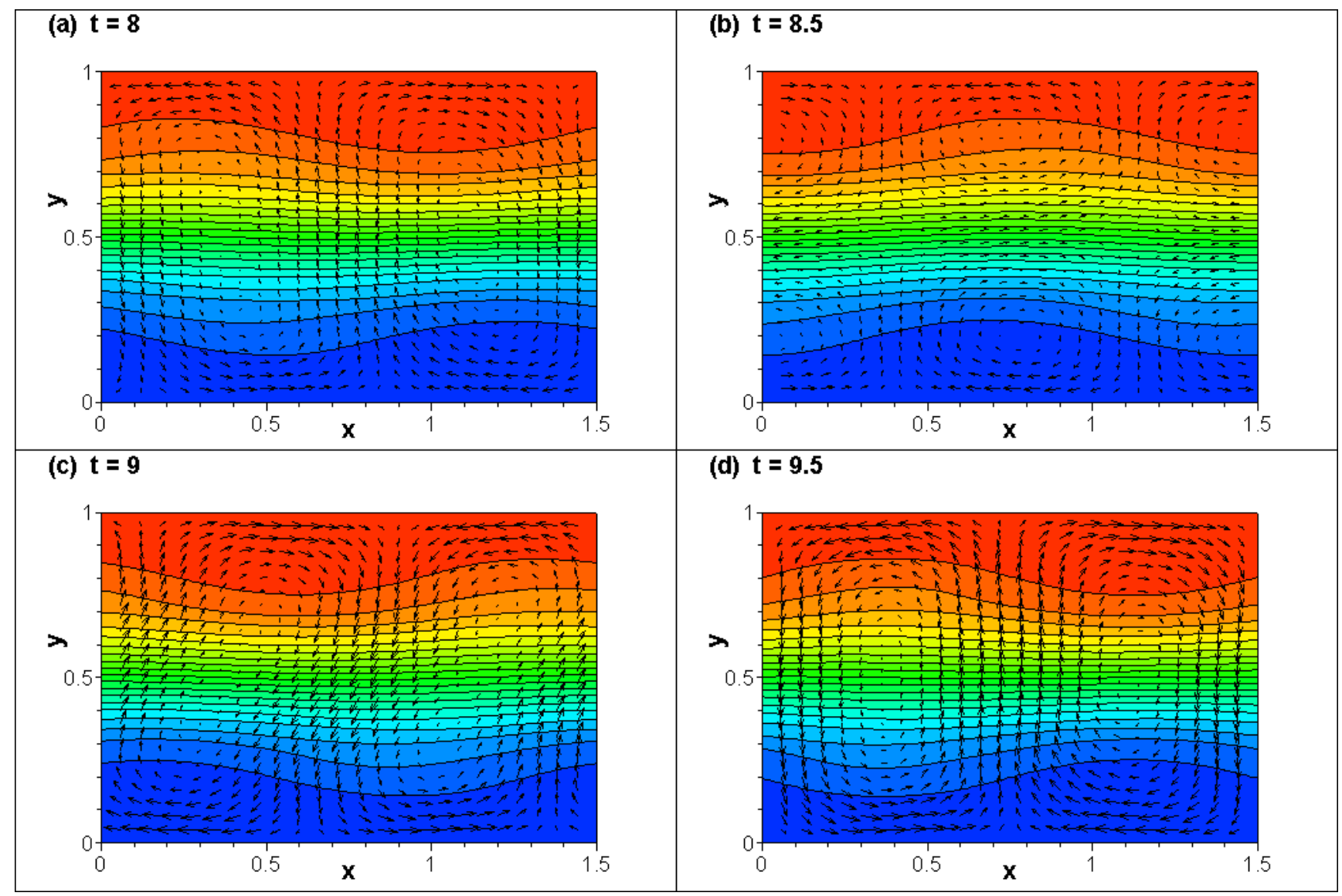

FIG. 4. Development of the Holmboe instability. The fields of concentration (isolines) and velocity (vectors) at different time moments. The data are obtained for $\delta=\delta_{U}=0.2, U_{0}=1, R e=100, C n=10^{-3}, P e=10^{6}, G r=10, k=4.19$, resolution $750 \times 500$ nodes.

the surface tension coefficient, which time changes are shown in figure $5 \mathrm{~d}$. One sees that the surface tension coefficient remains constant for the Holmboe instability, and this coefficient grows at the initial time moments for the Kelvin-Helmholtz instability. Finally, figures 5e,f show that the Kelvin-Helmholtz instability results in a stronger enhancement of the molecular mixing across the liquid/liquid boundary.

We also performed the numerical runs for the different values of the parameters, $U_{0}, P e, C n$. We found that the increase of the amplitude of the shear flow speeds up the instability. Lower Peclet numbers mean stronger diffusion effects, and the instability develops slower in this case owing to additional diffusive dissipation. The increase of the Cahn number increases the surface tension effect, which makes the interface less prone to deformations, and this reduces the amplitude of the waves and the amplitude of the hydrodynamic motion in the layer. Similar observations were earlier achieved with the help of the linear stability analysis [26].

The stability diagrams are depicted in figure 6 . The Kelvin-Helmhotz instability is always limited to lower Grashof numbers, while the evolution at higher Grashof numbers is dominated by the Holmboe instability. The zone of the Holmboe instability is large (in comparison to the zone of the Kelvin-Helmholtz instability), and remains unclosed from the top. Stronger viscous and diffusion effects should result in a closure of the zone of the Holmboe instability, although such cases are not modelled in the present work. Similar stability diagrams were earlier reported by other researchers $[11,12,26]$. In particular, figure $6 \mathrm{a}$ is adopted from our earlier work [26], where this diagram is obtained by using the linear stability analysis for an interface that separates two semiinfinite domains of two miscible liquids.

Figures $6 \mathrm{~b}-\mathrm{d}$ are obtained with the help of the numerical solution of the full equations. To perform the direct numerical simulations we assume that the liquids occupy a plane layer of finite height, that is used as a unit of length. The thickness of the velocity profile is taken as $\delta_{U}=0.2$. The thickness of the concentration profile was taken either equal to $\delta_{U}$ (figure $5 \mathrm{c}$ ) or five-times smaller than $\delta_{U}$ (figure $5 \mathrm{~b}, \mathrm{~d}$ ). In the linear stability theory the thickness of the velocity profile was used as a length scale. For correlation of these sets of data, in figures $6 \mathrm{~b}-\mathrm{d}$ we re-scale the wavenumber and Grashof number as $k \delta_{U}$ and $G r \delta_{U}$. The values of the other parameters should be also re-scaled as $P e \delta_{U}, \operatorname{Re} \delta_{U}$, and $C n / \delta_{U}^{2}$. 
(a)

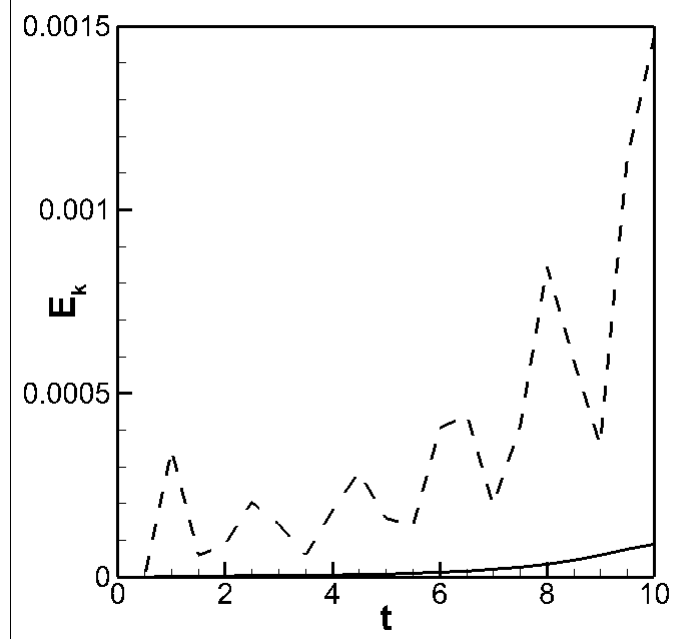

\section{(c)}

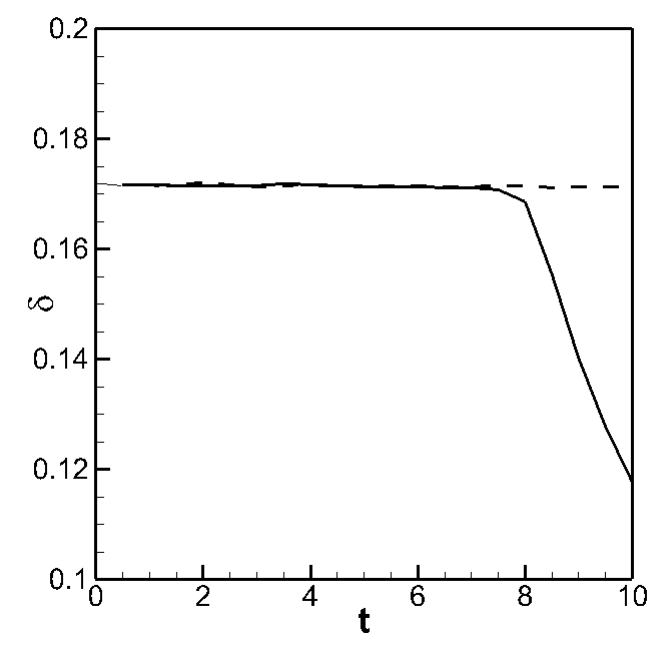

(e)

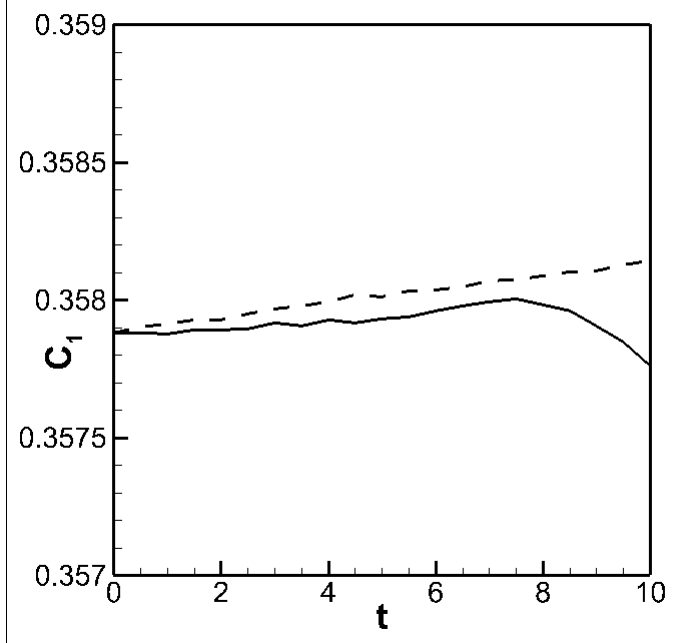

(b)

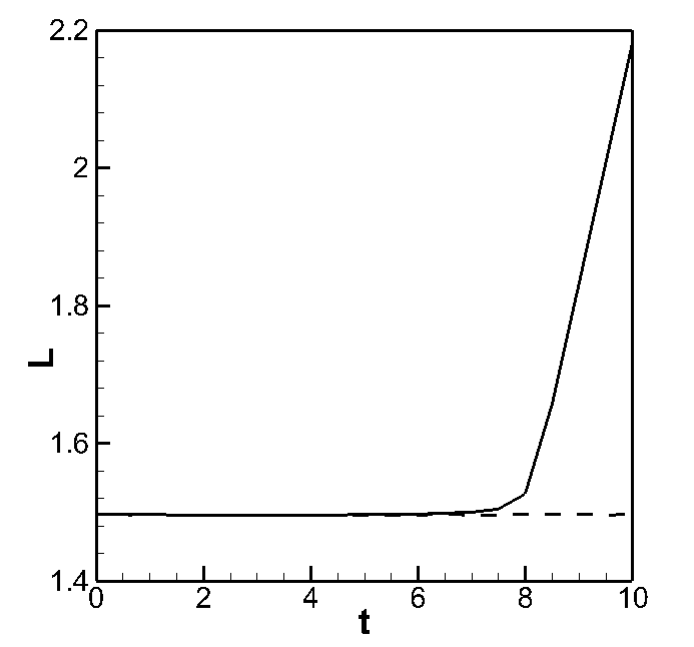

(d)

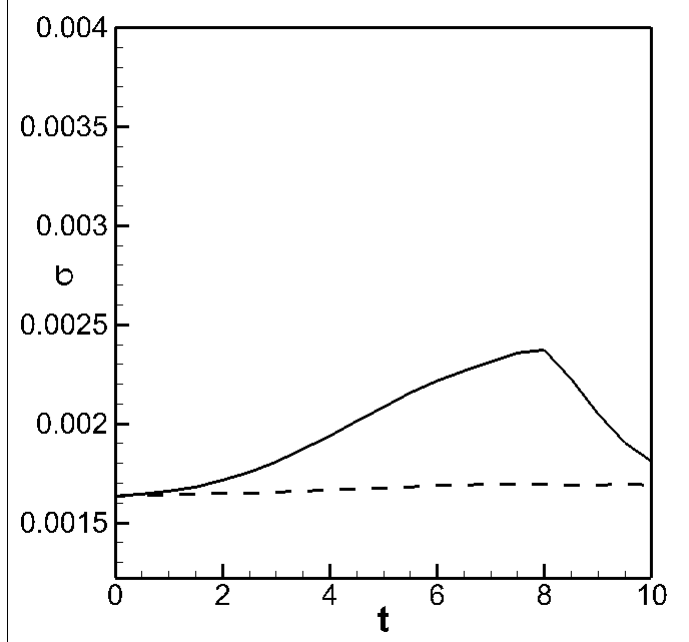

(f)

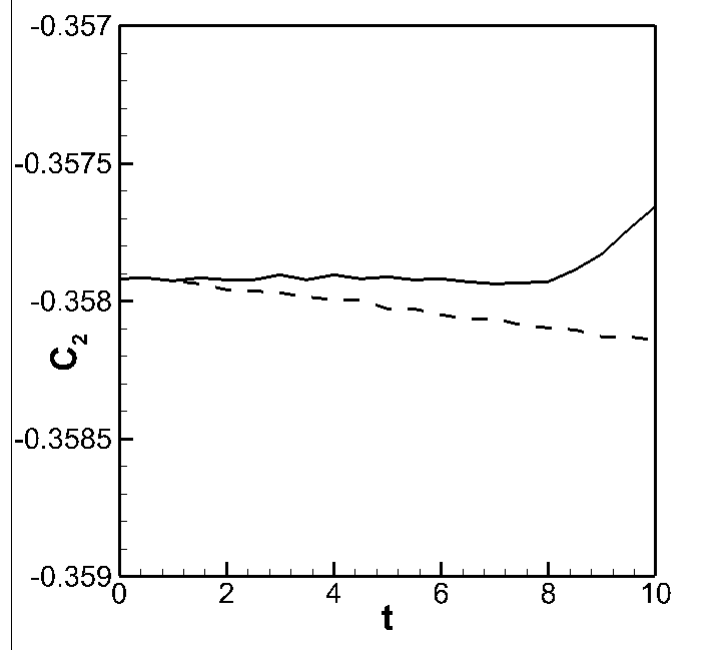

FIG. 5. (a) The total kinetic energy, (b) the thickness of the interface, (c) the length of the interface, (d) the surface tension coefficient, and (e,f) the average concentrations in each phase vs. time. The data are obtained for $\delta=\delta_{U}=0.2, U_{0}=1$, $R e=100, C n=0.001, P e=10^{6}, k=4.19, G r=0.5$ (solid line) and $G r=10$ (dashed line). 


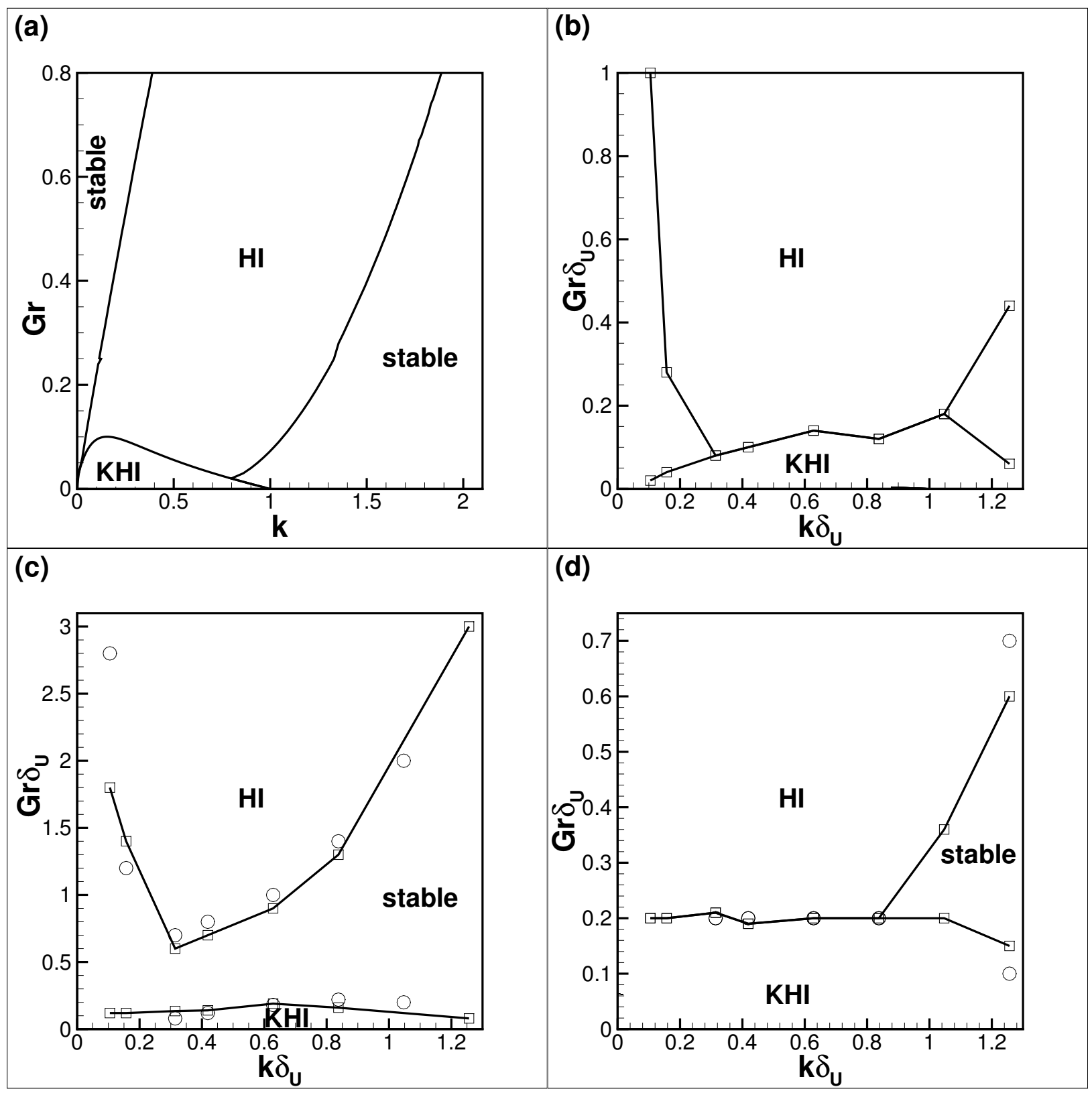

FIG. 6. The neutral curves defining the zones of the Kelvin-Helmoltz (marked by 'KHI') and Holmboe ('HI') instabilities. (a) The results of the linear stability analysis (see work [26] for more details) obtained for an immiscible interface $(P e=\infty)$ with no surface tension effects $(C n=0)$ that separates two semi-infinite inviscid liquid domains $(R e=\infty)$; other parameters are $U_{0}=1, \delta_{0}=0.2$. The thickness of the velocity profile, $\delta_{U}$, was used as a length scale. (b-d) The results of the direct numerical simulations. The data are obtained for $P e=10^{6}, R e=100, U_{0}=1$, and (b) $\delta_{0}=0.04, \delta_{U}=0.2, C n=4 \cdot 10^{-5}$; (c) $\delta_{0}=\delta_{U}=0.2, C n=0.001$; (d) $\delta_{0}=0.04, \delta_{U}=0.2, C n=0.001$. The unit of length is the height of the plane layer. The axis in (b-d) are re-scaled to simplify the comparisons with (a). Square symbols correspond to the phase-field simulations, and circles are the points obtained using the classical approach.

The new diagrams look very similar to the diagram obtained with the help of the linear stability theory. The addition of the surface tension effects extends the zones of the Kelvin-Helmholtz and Holmboe instabilities, as already noted in the linear stability study [26]. Although, we also identify a new behaviour, that was not observed in [26], when the zones of the Kelvin-Helmholtz and
Holmboe instabilities are separated by a zone of a stable shear flow (figure 6c): at lower Grashof numbers the shear flow is unstable to the Kelvin-Helmholtz instability, at higher Grashof numbers the shear flow develops the Holmboe instability, and there is a range of intermediate Grashof numbers when the shear flow is stable. In the linear study [26] the thickness of the interface was al- 
ways taken smaller than the thickness of the velocity profile, which is a generally expected relation for these parameters: the phase boundary is usually very thin (and, frequently, could be just several molecular layers), nevertheless, the interface thickness may be wider e.g. near a consolute point, when the behaviour depicted in figure $6 c$ can be realised.

Figures 6c,d also include the points (circles) that were obtained with the help of the classical approach, that completely disregards the surface tension forces and that models the diffusion process using the classical Fick's law (the diffusion flux is proportional to the gradient of concentration). In the classical approach there are no different phases, and instead, an evolution of a single-phase liquid with a impurity is studied. Namely, the governing equations for the classical approach read

$$
\begin{gathered}
\frac{\partial \vec{u}}{\partial t}+(\vec{u} \cdot \nabla) \vec{u}=-\nabla \Pi+\frac{1}{R e_{c}} \nabla^{2} \vec{u}-G r_{c} C \vec{\gamma} \\
\frac{\partial C}{\partial t}+(\vec{u} \cdot \nabla) C=\frac{1}{P e_{c}} \nabla^{2} C \\
\nabla \cdot \vec{u}=0 .
\end{gathered}
$$

These equations include three non-dimensional parameters,

$$
P e_{c}=\frac{L_{*} u_{*}}{D}, R e_{c}=\frac{\rho_{*} u_{*} L_{*}}{\eta_{*}}, G r_{c}=\frac{\phi g L_{*}}{u_{*}^{2}},
$$

the Peclet number, the Reynolds number, and the Grashof number.

In figure $6 \mathrm{c}, \mathrm{d}$ one sees that the majority of the 'classical' points lie very close to the phase-field results. This may be explained by the fact that too diffusive interfaces are studied in this work, so the surface tension forces remain low (the coefficient of the surface tension is reciprocal to the interface thickness $[26,39])$. The classical and phase-field approaches are based on the different laws for diffusion, but these differences remain inessential for determination of the boundaries of the hydrodynamic instabilities, as similar study is based on relatively shorter numerical runs when diffusive transport remains low (the changes in the average concentrations within each phase are small).

\section{CONCLUSIONS}

We study the isothermal evolution of a heterogeneous mixture of two slowly miscible liquids (with the heavier liquid underneath) enclosed in a horizontal plane layer. The shear flow is imposed along a miscible interface. With the help of the direct numerical simulation we investigate the development of the Kelvin-Helmotz and Holmboe instabilities of the shear flow.
In particular, we show that these two instabilities are characterised by a number of different distinctive features, which allow easy identification of the instability in each numerical run.

The development of the Kelvin-Helmholtz instability occurs through the formation of a flow vortex in the middle of the interface. The position of the vortex does not change with time. The kinetic energy associated with this vortex grows monotonically. The development of the Holmboe instability is associated with the formation of travelling waves on the interface, and with formation of moving vortices along the layer. In the case of Holmboe instability, the oscillatory growth of the kinetic energy is observed.

The identification of the instabilities help us to obtain the stability diagrams for a number of the sets of governing parameters. As expected the Kelvin-Helmholtz instability occurs at lower Grashof numbers (lower density contrasts of the liquids in contact), while the Holmboe instability occurs at higher Grashof numbers. For the parameters considered in this work, we could not find the level of the Grashof number when the zone of the Holmboe instability becomes closed from the top. In our simulations, even for very strong Grashof numbers, the shear flow is unstable. We however found a novel shape of the stability diagram, that has not been reported in earlier studies, e.g. in the linear stability study [26], when the zones of the Kelvin-Helmholtz and Holmboe instability are separated by an intermediate zone where the shear flow remains stable.

The numerical results are obtained on the basis of the phase-field approach. Some of the results (the stability diagrams) are also compared against the data obtained in the framework of the standard approach that models a binary mixture as a single-phase medium with a impurity. We found that for the determination of stability diagrams, the peculiarities of the phase-field approach turned out to be unimportant. There are two major differences of the phase-field approach from the classical model of miscible liquids, these are the account of the surface tension effects and the different treatment for the diffusion transport. The determination of the stability boundaries is based on the relatively short numerical runs. For similar runs, the interface remains almost flat, which makes the surface tension effects less significant. In addition, the Kelvin-Helmholtz and Holmboe instabilities develop on shorter (hydrodynamic) times when diffusion does not have time to impose any significant effect on the behaviour of a binary mixture. The features of the phase-field approach should become more pronounced for a longer evolution, at later stages of the instabilities.

\section{ACKNOWLEDGEMENTS}

The financial support of the Russian Federation for Basic Research (Grant No. 16-5110079), and the Royal Society (Ref. IE160277) is gratefully acknowledged. 
[1] L. Rayleigh, Proc. London Math. Soc. 1-11, 57 (1879).

[2] R. Fjortoft, Geofys. Pulb. Oslo 17, 1 (1950).

[3] S. Chandrasekhar, Hydrodynamics and Hydromagnetic Stability (Cambridge Press, 1961).

[4] P. G. Drazin, Introduction to hydrodynamic stability (Cambridge University Press, 2002).

[5] L. Howard, J. Fluid Mech. 10, 509 (1961).

[6] J. Holmboe, Geofys. Publ. 24, 67 (1962).

[7] P. G. Drazin, J. Fluid Mech. 4, 214 (1958).

[8] S. A. Maslowe and R. E. Kelly, J. Fluid Mech. 48, 405 (1971).

[9] P. Hazel, J. Fluid Mech. 51, 39 (1972).

[10] L. N. Howard and S. A. Maslowe, Boundary-Layer Met. 4, 511 (1973).

[11] P. G. Baines and H. Mitsudera, J. Fluid Mech. 276, 327 (1994)

[12] C. C. P. Caulfield, J. Fluid Mech. 258, 255 (1994).

[13] J. R. Carpenter, N. J. Balmfoth, and G. A. Lawrence, Phys. Fluids 22, 054104 (2010).

[14] G. A. Lawrence, S. P. Haigh, and Z. Zhu, Coastal and Estuarine Studies 54, 295 (1998).

[15] S. P. Haigha and G. A. Lawrence, Phys. Fluids 11, 1459 (1999).

[16] S. Alabduljalil and R. H. Rangel, J. of Engineering Math. 54, 99 (2006).

[17] A. Alexakis, Phys. Fluids 21, 054108 (2009).

[18] R. Barros and W. Choi, Phys. Fluids 23, 124 (2011).

[19] D. Koppel, J. Math. Physics 5, 963 (1964).

[20] W. D. Smyth and W. R. Peltier, J. Fluid Mech. 228, 387 (1991).

[21] S. A. Thorp, J. Fluid Mech. 32, 693 (1968).

[22] S. A. Thorp, Radio Science 4, 1327 (1969).
[23] A. M. Hoog and G. N. Ivey, J. Fluid Mech. 477, 339 (2003).

[24] D. D. Joseph and Y. Y. Renardy, Fundamentals of twofluid dynamics. Part II: lubricated transport, drops and miscible liquids (Springer-Verlag, 1993).

[25] A. Vorobev, Current Opinion in Colloid \& Interface Science 19, 300 (2014).

[26] A. Kheniene and A. Vorobev, Eur. Phys. J. E 38, 77 (2015).

[27] J. W. Cahn and J. E. Hilliard, J. Chem. Phys. 28, 258 (1958).

[28] J. Lowengrub and L. Truskinovsky, Proc. R. Soc. London, Ser. A 454, 2617 (1998).

[29] A. Vorobev, Phys. Rev. E 82, 056312 (2010).

[30] A. Vorobev and T. Lyubimova, J. Fluid Mech. 870, 543 (2019).

[31] L. Landau and E. Lifshitz, Statistical Physics, 3rd Edition, Part 1 (Pergamon Press, 1980).

[32] P. J. Flory, Principles of polymer chemistry (Cornell University Press, 1953).

[33] J. Pojman, C. Whitmore, M. Liveri, R. Lombardo, J. Marszalek, R. Parker, and B. Zoltowski, Langmuir 22, 2569 (2006).

[34] R. Xie and A. Vorobev, Journal of Colloid and Interface Science 464, 48 (2016).

[35] A. Vorobev and A. Boghi, Journal of Colloid and Interface Science 482, 193 (2016).

[36] A. Vorobev and E. Khlebnikova, Int. J. Heat Mass Trans. 125, 801 (2018).

[37] T. Lyubimova, A. Vorobev, and S. Prokopev, Phys. Fluids 31, 014104 (2019).

[38] A. Vorobev and T. Lyubimova, J. Fluid Mech. 870, 563 (2019).

[39] A. Kheniene and A. Vorobev, Phys. Rev. E 88, 022404 (2013). 